\title{
Rôle de la charge inorganique des dentifrices sur la formation des biofilms
}

\author{
Frank Hui, Éric PALAYODAN, Benoît QUILICHINI et Jean LÉDION* \\ Arts et Métiers - Paris Tech, LIM-UMR CNRS 8006, 151 boulevard de l'Hôpital, \\ 75013 Paris, France \\ *Auteur de correspondance : jean.ledion@ensam.eu
}

Résumé - Les biofilms jouent un rôle important dans le développement de micro-organismes très divers, que ce soit dans l'eau destinée à la consommation humaine, dans les eaux grises ou tout simplement dans les ressources naturelles. Or, à la suite de nombreux travaux effectués sur plusieurs centaines de biofilms, on a pu prouver, sans conteste, que tous les biofilms étudiés contenaient des produits minéraux en quantité quelquefois très importantes. Ces produits minéraux sont caractérisables (formule, cristallinité) grâce à la technique d'analyse par spectrométrie d'absorption infrarouge sur micropastilles. Ces divers produits sont mesurables, soit directement par une absorbance caractéristique, soit en comparant les absorbances avant et après calcination de la micropastille, la matière organique étant ainsi éliminée. C'est pour cela que l'on s'est intéressé plus particulièrement aux biofilms formés dans les évacuations de l'eau usée des lavabos à usage domestique, à cause de leur abondance remarquable, liée à une vitesse de développement très rapide. En mettant en œuvre les méthodes d'analyse précitées, on a pu ainsi prouver que c'était la charge minérale des dentifrices qui favorisait le développement de ces biofilms.

Mots-clés: Infrarouge, dentifrice, biofilms, composés minéraux

Abstract - Role of inorganic products with toothpaste on the biofilm
development. Biofilms have a significant effect on the development of
numerous micro-organisms, as well in the case of waters intended
for human consumption, as in the case of greywaters or natural resources.
However, on the base of many analyses carried out on hundreds of
biofilms, it was proven without any doubt that all the studied biofilms contain
inorganic products sometimes in significant quantity. The characteristics of
these inorganic products (formula, crystallinity) can be determined using
the infrared absorption spectrometry based on CsBr micro-pellet analysis.
These various products are analysed semi-quantitatively either directly
by the measure of their absorbance or by comparing the absorbance 
before and after micro-pellet calcination. The calcination leads to the elimination of the organic compounds. The results show that the inorganic products are particularly abundant in the biofilms developed in the wastewater drains of domestic wash-hand basins, because of their remarkable abundance, due to very fast development kinetic. Thanks to the analysis methods, it is proven that silicon inorganic charges of the toothpastes are the main source of the development of these biofilms. In the case of non-domestic wash-hand basins, without toothpaste use, the biofilm development kinetic is lower.

Keywords: Infrared spectroscopy, toothpaste, biofilms, inorganic compounds

\section{INTRODUCTION}

Pour la plupart des auteurs, le biofilm est considéré sous son aspect « organique ». Par exemple, les pionniers en la matière (Costerton, 2004) définissent le biofilm comme une " organisation structurée de micro-organismes dans une matrice polysaccharidique et protéique, protectrice et nutritive, adhérant à une surface ».

Selon eux, dans le cas le plus simple, ce biofilm serait composé de cellules bactériennes et de leurs métabolites. Un tel biofilm aurait généralement une structure très poreuse (plus de $80 \%$ d'eau), et comporterait également une part indéterminée de particules inorganiques piégées dans des exopolymères. De plus, au sein d'un biofilm, des organismes plus évolués, comme des algues, des protozoaires, cohabiteraient avec les bactéries. . .

Les matières inorganiques ne sont citées qu'en passant, sans doute dans le souci d'être complet et de ne rien oublier dans l'énumération, et ceci pour bien souligner la complexité du système. Cependant, d'autres auteurs se sont tout de même intéressés aux relations minéraux/bactéries (Thomassin et al., 2002; Hiernaux, 2005). Ces contributions restent, malgré tout, relativement peu nombreuses.

Ces biofilms peuvent être très « discrets » et quasi-invisibles, l'observateur ne les décelant que par l'aspect gras ou gluant qu'ils peuvent donner aux surfaces. Mais il en existe d'autres qui se développent de manière invasive, allant jusqu'à envahir toute une installation. En ce qui concerne les équipements sanitaires des logements, individuels ou collectifs, on peut constater, par simple observation, que ce sont les lavabos qui contribuent le plus à former des biofilms au niveau de l'évacuation de l'eau usée. Les autres équipements sanitaires, de cuisine ou de salle de bain, ne donnent que des biofilms en quantité beaucoup plus réduite.

\section{MATÉRIELS ET MÉTHODES}

\subsection{Eau utilisée}

Les eaux intervenant dans la formation des biofilms étudiés (Eaux de Paris et Marseille notamment) n'ont pas fait l'objet d'une caractérisation particulière. Il s'agit d'eaux très classiques, généralement bicarbonatées-calciques, plus ou 
moins minéralisées, dont le rôle est généralement secondaire dans la formation des biofilms.

\subsection{Méthode d'analyse}

La spectrométrie d'absorption infrarouge, utilisée dans cette étude, est une méthode aujourd'hui très classique et largement utilisée en routine par de nombreux laboratoires. Bien qu'elle soit ancienne, elle a connu un regain d'intérêt avec l'apparition d'appareils utilisant la transformée de Fourier. Cependant, les applications se cantonnent souvent à la chimie organique, alors qu'elle est, en général, nettement sous-utilisée pour l'analyse des produits inorganiques, même si certains pionniers, comme Lecomte en avait décrit, au milieu du siècle dernier, et dans le détail, l'intérêt pour les substances aussi bien organiques qu'inorganiques (Lecomte, 1958).

De nos jours, l'obtention de spectres complets est beaucoup plus rapide, fournissant des informations sur l'hydratation des produits, les différentes matières organiques et les divers types de composés inorganiques présents. Bien sûr, la méthode a aussi des limites, mais elles ne sont généralement pas contraignantes pour l'étude de la fraction minérale des biofilms.

La spectrométrie infrarouge ne fait, elle, en principe, aucune différence entre composés organiques et inorganiques. Elle voit seulement les mouvements (vibrations par exemple) des atomes, les uns par rapport aux autres. Sur la figure 1, on voit l'aspect d'un spectre qui montre à la fois de la matière organique et des produits minéraux. L'important est que les informations concernant les divers types de composés se trouvent être séparées, la fréquence dépendant de la masse des atomes aussi bien que de la longueur des liaisons. Ces informations peuvent être traitées indépendamment. De manière générale, la masse des groupements d'atomes, à l'origine d'une bande, augmente de l'infrarouge proche à l'infrarouge lointain, c'est-à-dire de $\lambda=2,5 \mu \mathrm{m}$ à $50 \mu \mathrm{m}$ en longueur d'onde, ou encore, en prenant $1 / \lambda$, de 4000 à $200 \mathrm{~cm}^{-1}$ en nombre d'ondes $\left(v^{*}\right)$.

Schématiquement, il est connu que les groupements $\mathrm{O}-\mathrm{H}$ sont les premiers à apparaître entre 4000 et $3000 \mathrm{~cm}^{-1}$, suivis des liaisons organiques (surtout entre 3000 et $1000 \mathrm{~cm}^{-1}$ ), puis, pour les composés inorganiques, les groupements $\mathrm{CO}_{3}, \mathrm{SO}_{4}$, $\mathrm{SiO}_{4}$, de 1550 à $600 \mathrm{~cm}^{-1}$ et enfin les oxydes et oxyhydroxydes métalliques entre 600 et $200 \mathrm{~cm}^{-1}$.

Par ailleurs, comme cette technique prend en compte la symétrie des groupements d'atomes et de la maille cristalline, elle permet de distinguer les variétés allotropiques d'un même composé chimique. Par exemple, le carbonate de calcium $\mathrm{CaCO}_{3}$ donne des bandes d'absorption différentes selon qu'il est sous une forme amorphe et très hydratée ou, plus classiquement, sous forme de calcite, d'aragonite ou de vatérite.

Au-delà d'une approche qualitative, des mesures quantitatives sont possibles. C'est pourquoi il est intéressant de pouvoir mesurer les quantités de matières prélevées. L'information se trouve inscrite sur les documents sortant du spectromètre, mais il faut savoir l'en extraire avec certitude. Le principe est simple: après broyage de l'échantillon dans du bromure de césium (transparent à l'infrarouge) servant de dispersant, on comprime l'ensemble sous forme d'une pastille dont l'aire présentée 
au spectromètre est constante. Dans ces conditions, les absorbances sont additives et, pour un groupement donné, l'absorbance $A$ est proportionnelle à la masse $m$ du groupement présent dans la pastille. La pratique est un peu plus complexe. Elle dépend notamment de la qualité des spectres obtenus (Nyquist et Kagel, 1971; Labbé et al., 2008).

\subsection{Prélèvement et pastillage}

Pour obtenir un spectre représentatif du biofilm étudié, il est nécessaire de faire le prélèvement de manière rigoureuse. La première opération consiste à procéder à un lavage du biofilm à l'eau déminéralisée, de manière à éliminer au maximum l'eau du réseau qui l'imbibe et les « sels » qu'elle contient. Un séchage modéré, par évaporation à la température ambiante (ou éventuellement à l'étuve jusqu'à $60{ }^{\circ} \mathrm{C}$ maximum), suffit alors à éliminer l'excès d'humidité sans dénaturer le produit à analyser. La seconde opération est le prélèvement proprement dit. Lorsque le biofilm est abondant, on peut utiliser une technique de micro-abrasion à l'aide de petits outils en acier inoxydable. S'il est invisible à l'œil nu, on procède par contact : du bromure de césium en poudre fine est frotté sur la surface et se pollue par le biofilm. C'est ce bromure enrichi en biofilm qui sera analysé.

On procède alors au pesage (en routine, une pesée de 10 à $100 \mu \mathrm{g}$ ) à l'aide d'une balance automatique au $\mu \mathrm{g}$. Le broyage doit être soigné, d'une durée standardisée (10 min par exemple). Il se fait en présence de bromure de césium (23 $\pm 2 \mathrm{mg}$ pour une pastille de $5 \mathrm{~mm}$ de diamètre). Ce dernier est indispensable pour obtenir des spectres dans l'infrarouge lointain (jusqu'à $200 \mathrm{~cm}^{-1}$ ). L'opération se fait dans un mini-mortier en alumine monocristalline $\left(\mathrm{a}-\mathrm{Al}_{2} \mathrm{O}_{3}\right)$, généralement incolore. Après broyage, on procède immédiatement au pastillage à l'aide d'un moule approprié (5 ou $3 \mathrm{~mm}$ de diamètre) placé sous une presse hydraulique.

\subsection{Spectres infrarouges}

Les pastilles sont analysées avec un spectromètre Perkin-Elmer à transformée de Fourier, avec une résolution de 4 ou $8 \mathrm{~cm}^{-1}$ en accumulant 120 spectres (conditions de routine). Tous les spectres sont enregistrés en mode absorbance, donnant ainsi une échelle linéaire pour les mesures quantitatives. L'appareil est équipé d'un système de réduction du trajet optique dans l'air, afin de minimiser les perturbations liées à l'air ambiant (humidité, $\mathrm{CO}_{2}$ ). La figure 1 donne un exemple de spectre ainsi obtenu sur un biofilm formé à partir d'eau potable.

Sur ce spectre, formé sur une surface en polyéthylène, on observe bien l'eau résiduelle d'hydratation du biofilm $(\mathrm{O}-\mathrm{H})$, les bandes (liaisons $\mathrm{C}-\mathrm{H}$, vers $2950 \mathrm{~cm}^{-1}$ ) des matières organiques et les bandes amides I et II des protéines, caractéristiques d'une activité biologique, ainsi que la présence de minéraux : carbonate de calcium sous forme de calcite (repérée C) et aluminosilicates (repérés $\mathrm{SiO}_{4}$ ). 


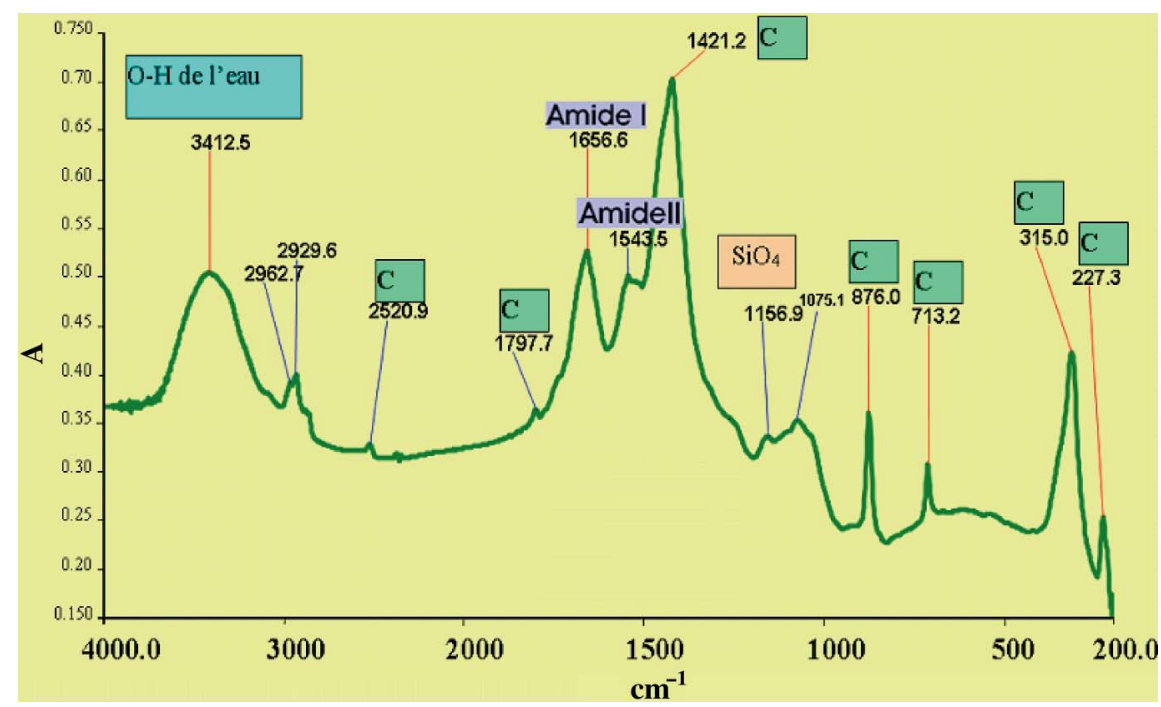

Fig. 1. Spectre d'un biofilm (sans précaution particulière de prélèvement).

Fig. 1. Biofilm spectrum (without particular caution).

\subsection{Opérations complémentaires}

Le dépouillement des spectres obtenus peut se heurter à diverses difficultés lorsqu'il y a interférence entre les bandes d'absorption de plusieurs produits. C'est pourquoi on peut, après enregistrement du spectre, rebroyer la pastille, puis la calciner à une température appropriée. Cette calcination peut avoir pour objet la transformation d'une substance, peu visible en IR, en une autre qui absorbe avec plus de netteté (par exemple, les sulfures qui peuvent être transformés en sulfates), ou bien l'élimination totale des matières organiques par calcination à $550{ }^{\circ} \mathrm{C}$ en atmosphère oxydante.

Après calcination, on reconstitue une pastille qui est passée dans les mêmes conditions dans le spectromètre. La perte de substance, lors des transferts, est limitée à $1 \%$ si l'on prend soin de piéger les résidus sur les parois (de la coupelle par exemple), avec très peu de $\mathrm{CsBr}$ (pur, broyé), en les ajoutant à la pastille. On peut voir, sur la figure 2 , le spectre du résidu minéral, après calcination à $550{ }^{\circ} \mathrm{C}$ pendant $30 \mathrm{~min}$, du biofilm de la figure 1 . On retrouve de l'eau en faible quantité (eau atmosphérique reprise par hygroscopie lors du pastillage), de la calcite (notée C), ainsi que des aluminosilicates (notés $\mathrm{SiO}_{4}$ ).

\subsection{Caractérisation des micro-organismes}

De manière parallèle, des recherches systématiques de la nature de la flore bactérienne de ces mêmes biofilms peuvent être mises en œuvre. Le biofilm est décroché par grattage, écouvillonnage ou passage dans une cuve à ultrasons. Dans la 


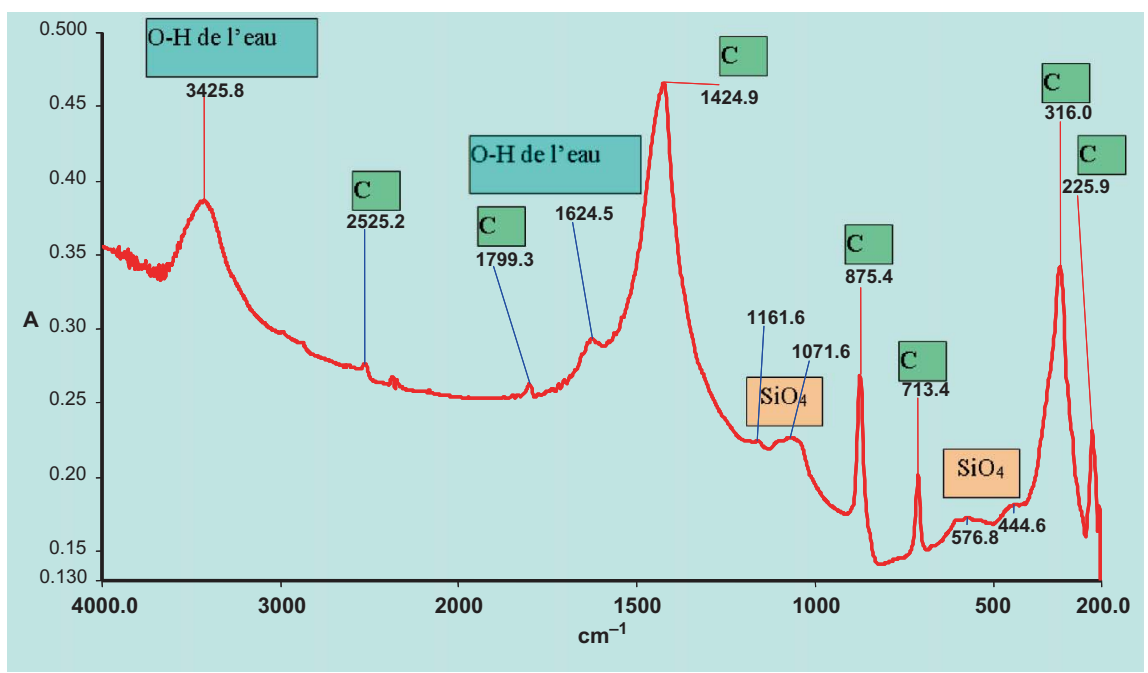

Fig. 2. Spectre du biofilm de la figure 1 , après calcination à $550{ }^{\circ} \mathrm{C}$ durant $30 \mathrm{~min}$.

Fig. 2. Biofilm spectrum of figure 1 , after calcination at $550^{\circ} \mathrm{C}$ for $30 \mathrm{~min}$.

présente étude, on s'est limité à la caractérisation de la fraction minérale des biofilms analysés.

\section{RÉSULTATS OBTENUS SUR DEUX BIOFILMS CARACTÉRISTIQUES}

Parmi les divers biofilms recueillis, on a étudié en détail les prélèvements ci-dessous :

\begin{tabular}{|lccc|}
\hline & Lieu & Utilisation lavabo & Apparence \\
\hline Biofilm $n^{\circ} 1$ & Paris & 2 personnes & Dépôt noir \\
Biofilm $n^{\circ} 2$ & Marseille & 6 personnes & Dépôt noir \\
\hline
\end{tabular}

Ces deux types de biofilm sont donc formés avec des eaux différentes, dans des logements où les modes de vie sont différents. Par ailleurs, les produits d'hygiène utilisés sont également différents sur les deux sites.

\subsection{Biofilm $n^{\circ} 1$}

Sur la figure 3, on a reporté le spectre infrarouge du premier biofilm observé. On constate qu'il a bien toutes les caractéristiques d'un biofilm classique. L'eau est 


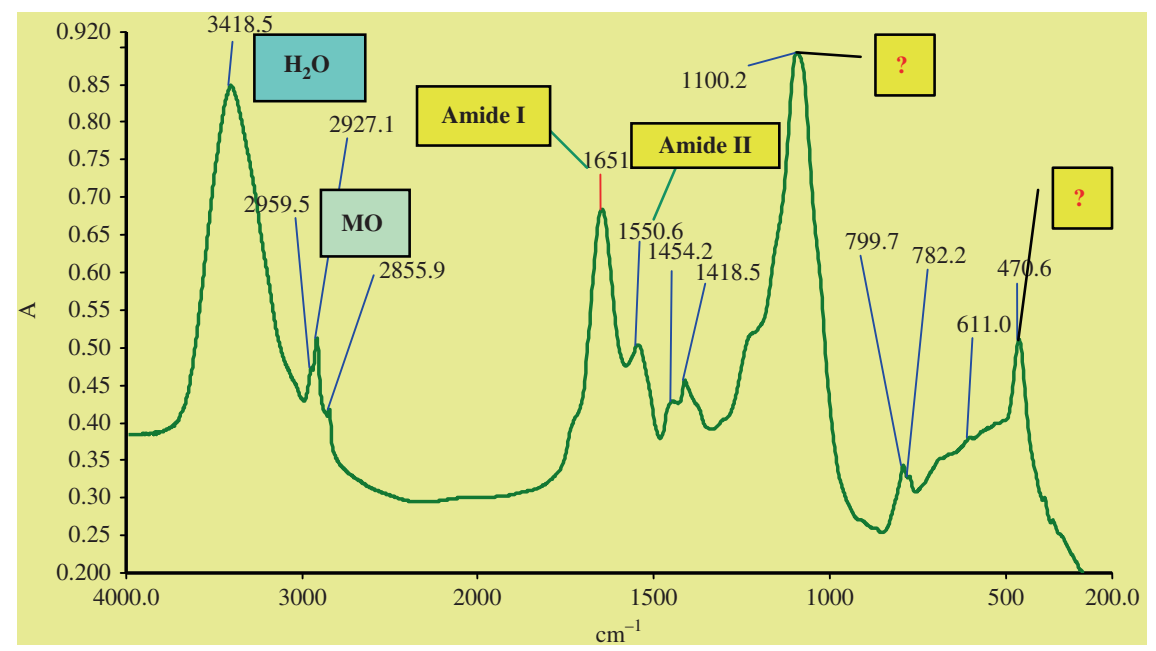

Fig. 3. Spectre IR du biofilm $\mathrm{n}^{\circ} 1$ formé au niveau de l'évacuation du lavabo.

Fig. 3. IR spectrum of the biofilm $n^{\circ} 1$ provided from the wash-hand basin drain.

abondante (malgré un séchage préalable du prélèvement de 30 min à $60{ }^{\circ} \mathrm{C}$ ), elle apparaît au niveau des liaisons $\mathrm{OH}$ à $2419 \mathrm{~cm}^{-1}$. Les matières organiques apparaissent au niveau des liaisons C-H (zone des $2900 \mathrm{~cm}^{-1}$ ), mais aussi des bandes amide I et amide II $\left(1652\right.$ et $\left.1551 \mathrm{~cm}^{-1}\right)$. Ce sont ces bandes d'absorption qui sont les plus caractéristiques des biofilms. Par ailleurs, deux bandes importantes, non identifiées, apparaissent vers 1100 et $470 \mathrm{~cm}^{-1}$, dans le domaine des phosphates et/ ou silicates. Après avoir effectué une calcination de $30 \mathrm{~min}$ à $550^{\circ} \mathrm{C}$, on obtient le spectre de la figure 4 . On constate que les matières organiques ont bien disparu. Il reste un peu d'eau provenant de la reprise d'eau atmosphérique par la pastille (à 3420 et $1626 \mathrm{~cm}^{-1}$ ). Les deux massifs à 1099 et $468 \mathrm{~cm}^{-1}$, observés avant calcination, apparaissent quasi-inchangés. On confirme donc la présence de silicates et/ou phosphates. Comme ces produits sont en quantité importante, on peut penser qu'ils ne proviennent pas tous de l'eau du réseau parisien où ils ne sont présents qu'en quantité très faible.

\subsection{Biofilm $n^{\circ} 2$}

Les spectres IR obtenus pour ce biofilm (fig. 5) montrent une grande analogie avec ceux obtenus précédemment, bien que le site soit différent (Marseille au lieu de Paris) et que la population concernée soit différente. La calcination laisse apparaître les mêmes résidus, qui là encore ne peuvent s'expliquer par les MES des eaux distribuées. D'autres biofilms, issus de diverses résidences de la région parisienne, ont été analysés et ont donné des spectres semblables à ceux obtenus pour le biofilm $n^{\circ} 1$.

En conséquence, on peut penser que la qualité de l'eau utilisée n'est pas le facteur déterminant dans la formation de ces biofilms. Il est donc nécessaire de s'intéresser 


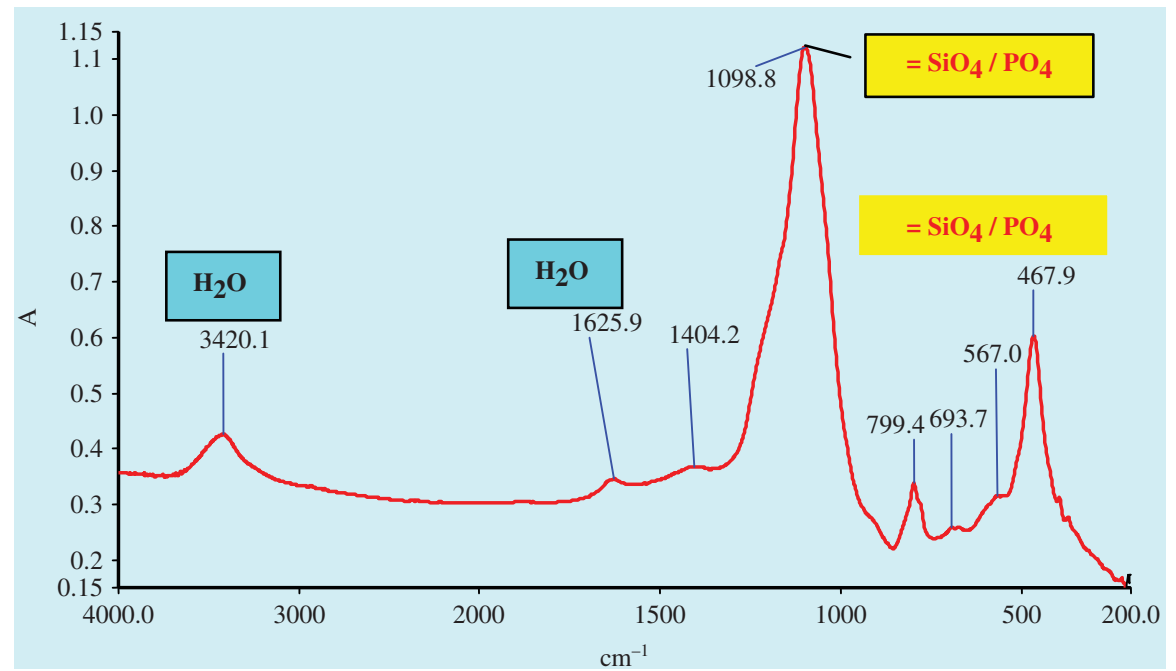

Fig. 4. Spectre IR, après calcination de $30 \min$ à $550{ }^{\circ} \mathrm{C}$ du biofilm $\mathrm{n}^{\circ} 1$.

Fig. 4. IR spectrum of the biofilm $n^{\circ} 1$, after calcination at $550^{\circ} \mathrm{C}$ for $30 \mathrm{~min}$.

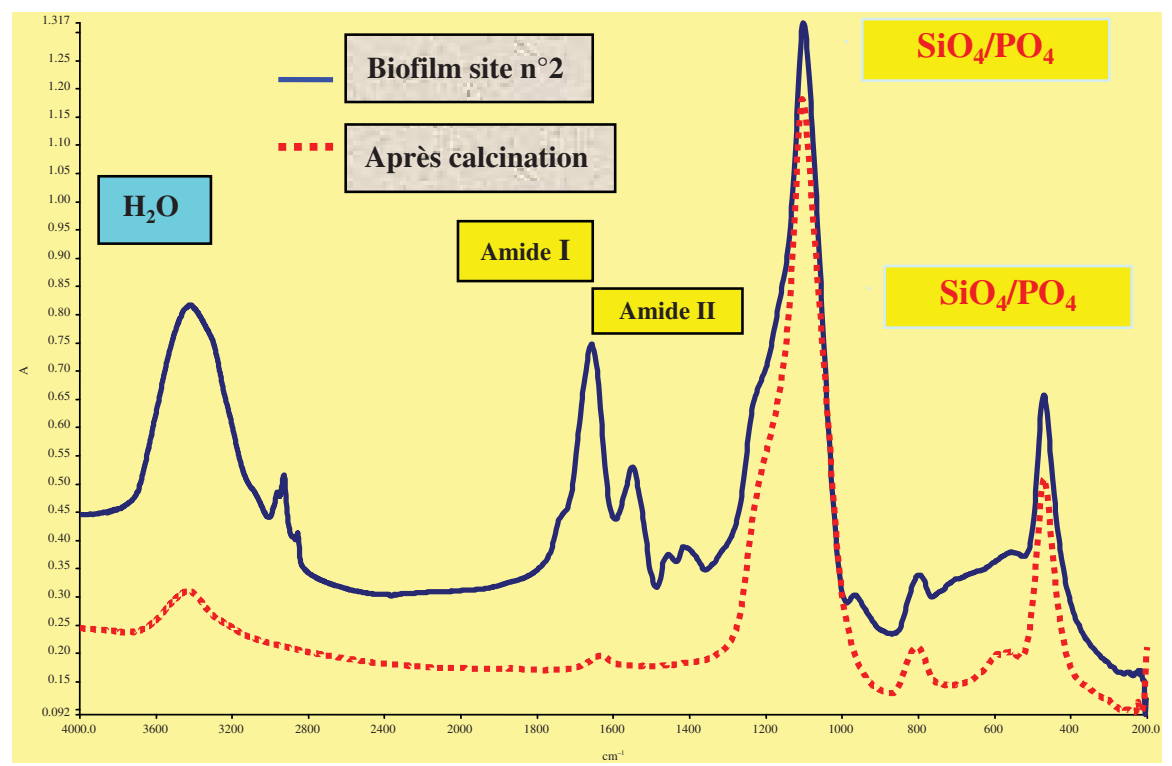

Fig. 5. Spectres IR, avant et après calcination, du biofilm $n^{\circ} 2$ (évacuation de lavabo). Fig. 5. IR spectra of the biofilm $n^{\circ} 2$ provided from the wash-hand basin drain, before and after calcination at $550^{\circ} \mathrm{C}$ for $30 \mathrm{~min}$. 


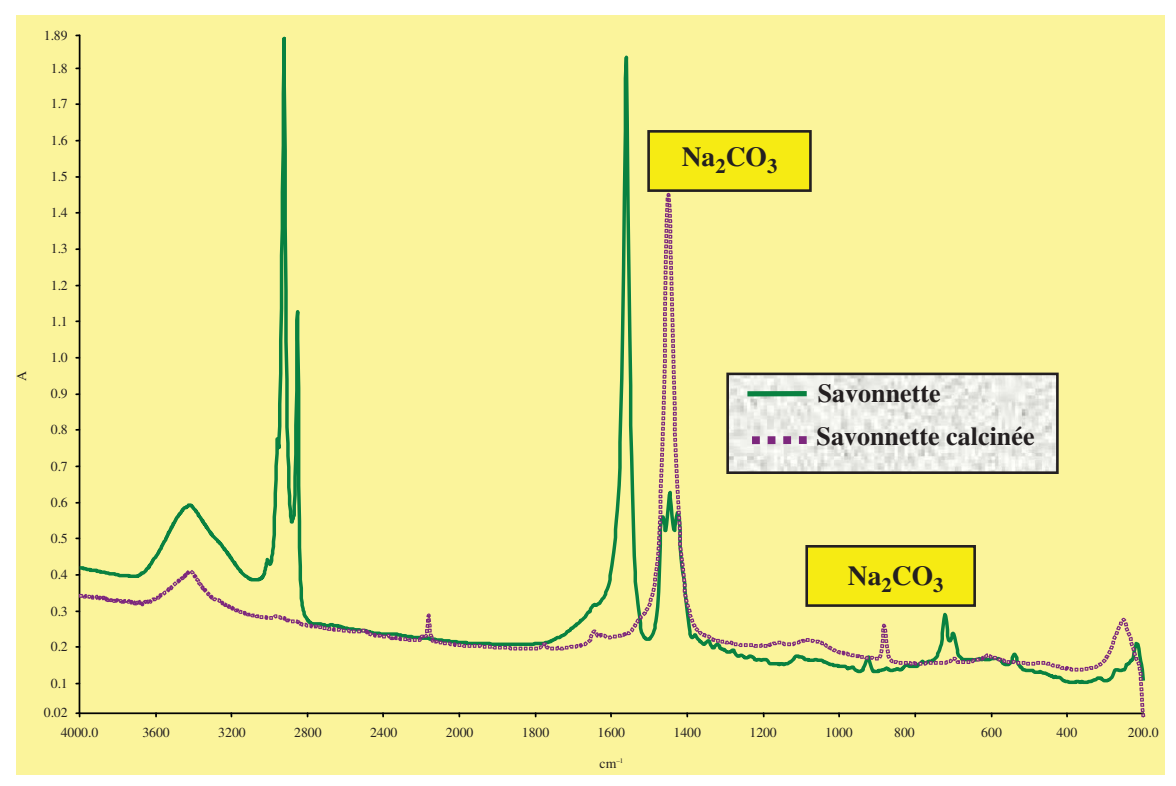

Fig. 6. Spectres IR, avant et après calcination, d'un savon utilisé sur le site $\mathrm{n}^{\circ} 1$.

Fig. 6. IR spectra, before and after calcination, of a soap used in the site $n^{\circ} 1$.

alors aux produits d'hygiène utilisés quotidiennement par les habitants concernés. Ces produits font essentiellement partie de la catégorie des savons et des dentifrices.

\subsection{Caractérisation IR des savons}

À titre d'exemple on a reporté, sur la figure 6, les spectres, avant et après calcination, d'une savonnette d'usage courant. On voit, qu'après élimination des matières organiques par calcination à $550{ }^{\circ} \mathrm{C}$, qu'il ne reste que deux pics caractéristiques qui correspondent au carbonate de sodium anhydre $\mathrm{Na}_{2} \mathrm{CO}_{3}$. $\mathrm{Ce}$ produit étant absent des spectres des biofilms étudiés, ce ne sont donc pas les savons qui fournissent la charge minérale. C'est ce qui conduit à examiner quelle est la charge minérale des dentifrices.

\subsection{Caractérisation IR des dentifrices}

Pour le site $\mathrm{n}^{\circ} 1$, on a effectué une comparaison entre la charge inorganique du biofilm et celle du dentifrice habituellement utilisé sur le site. C'est ce que l'on peut voir sur la figure 7. Contrairement à ce qui avait été observé pour les savons, il y a ici une très grande analogie entre les deux spectres.

On a donc effectué, pour vérifier le caractère général de ce qui avait été observé, des spectres de comparaison entre divers dentifrices. La figure 8 indique sans ambages que les charges de la plupart des dentifrices sont identiques : il s'agit, 


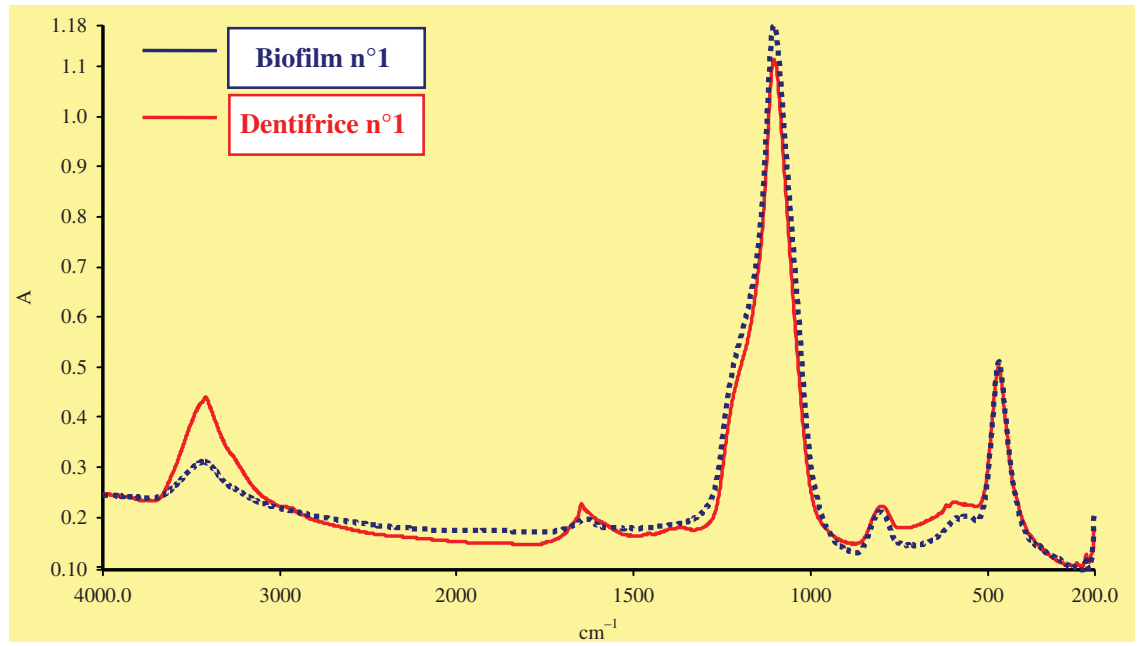

Fig. 7. Spectres IR, après calcination de $30 \mathrm{~min}$ à $550^{\circ} \mathrm{C}$, du biofilm et du dentifrice utilisé, site $n^{\circ} 1$.

Fig. 7. IR spectra, after calcination at $550^{\circ} \mathrm{C}$ for $30 \mathrm{~min}$, of the biofilm and the toothpaste used in site $n^{\circ} 1$.

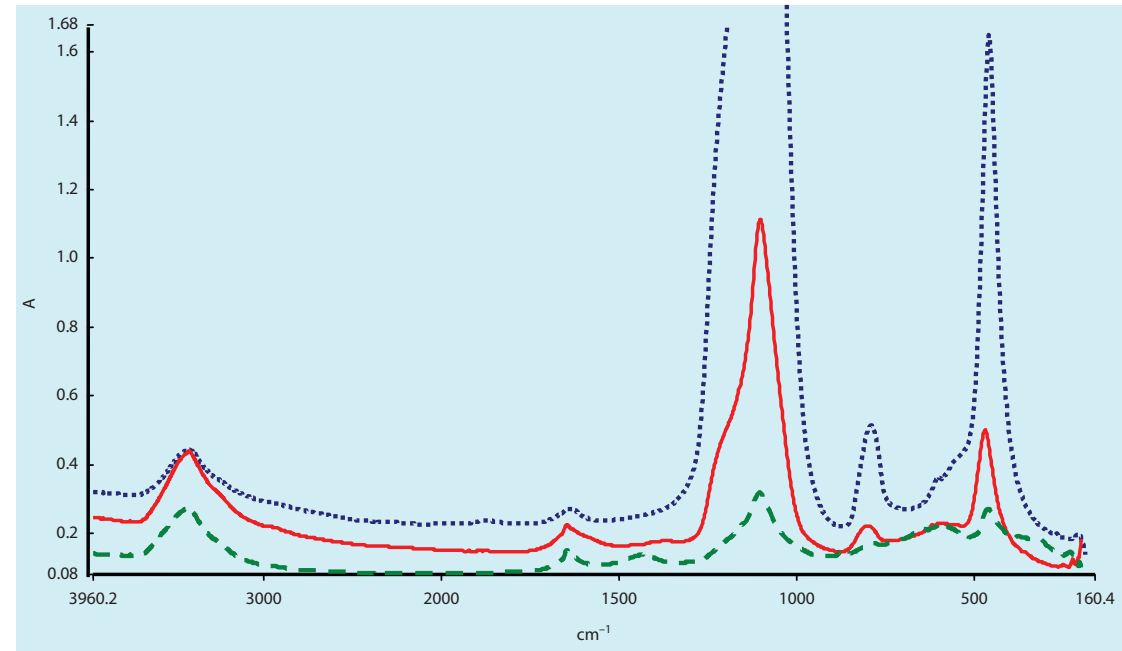

Fig. 8. Spectres IR, après calcination, de trois dentifrices différents.

Fig. 8. IR spectra, after calcination, of three different toothpastes. 


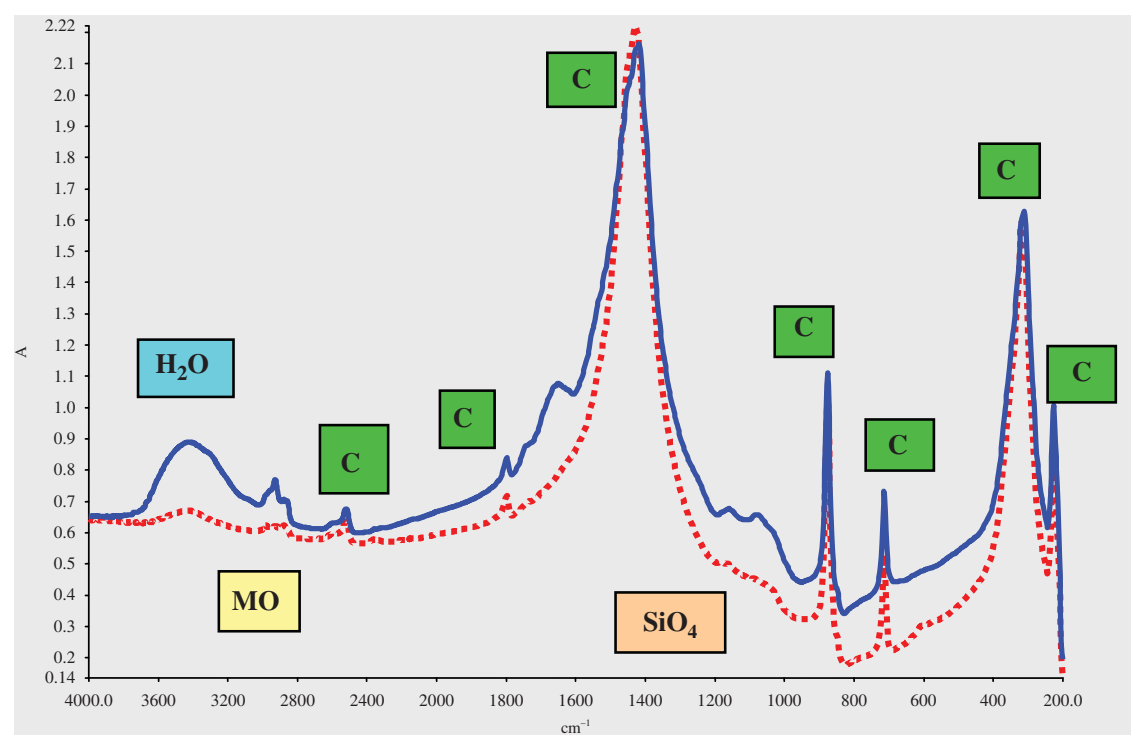

Fig. 9. Spectres IR, avant et après calcination, de biofilm de lavabo sans usage de dentifrice. Fig. 9. IR spectra, before and after calcination, of biofilm of wash-hand basin drain without toothpaste use.

selon les fabricants de « silices amorphes », ce qui peut expliquer les bandes d'absorption en infrarouge correspondant aux liaisons silicium-oxygène. Néanmoins, on ne peut pas exclure la présence de silicates et de phosphates qui absorbent pour les mêmes nombres d'onde.

\subsection{Biofilm sans dentifrice}

Afin de vérifier le bien-fondé du rôle des dentifrices dans la formation des biofilms de lavabo, on a procédé également à l'analyse, par spectrométrie IR, de biofilms formés dans des lavabos situés dans des immeubles de bureaux où, normalement, l'usage des dentifrices est plus limité. Un exemple est donné sur la figure 9.

On observe que, dans ce cas, les aluminosilicates et/ou phosphates sont bien présents (repérés $\mathrm{SiO}_{4}$ sur les spectres), mais en quantité beaucoup plus limitée. Il en est de même pour les matières organiques (repérées MO). Le produit dominant est le carbonate de calcium (repéré C) sous la forme de calcite ; ce carbonate étant absent des biofilms formés en présence de dentifrice avec le même type d'eau.

\section{DISCUSSION}

En matière de biofilm, les analyses effectuées, après calcination, ont toujours prouvé sans conteste que ceux-ci contiennent toujours (Hui et al., 2010) des produits 
minéraux qui sont caractérisables (formule, cristallinité) et mesurables, soit directement par une absorbance caractéristique, soit en comparant les absorbances avant et après calcination. Il est important de constater que, par exemple, sur environ 150 biofilms formés à partir d'eaux destinées à la consommation humaine et analysées par nos soins, on trouve toujours des quantités importantes d'aluminosilicates. Or, ces eaux sont des eaux de faible turbidité (inférieure en général à 0,2 NTU). On peut donc penser qu'il existe une synergie entre micro-organismes et particules colloïdales d'argile dans le développement des biofilms. Il faut alors considérer que les substances minérales sont autre chose que des « impuretés » piégées par le biofilm lors de sa constitution. Au contraire, elles sont réellement constitutives de ce biofilm. Dans le cas présent, nous avons déjà vu que les quantités des deux composés majoritaires (organique et minéral) ne sont pas d'un ordre de grandeur comparable. La composante minérale (qui absorbe peu dans l'infrarouge) est bien plus importante que la partie organique (qui absorbe fortement dans l'infrarouge).

En effet, dans une étude déjà ancienne, qui associait la spectrométrie infrarouge avec d'autres techniques telles que la spectrométrie de photoélectrons X, les auteurs avaient pu conclure à une interaction protéines-phosphates-calcium (Daufin et Labbé, 1998), en milieu lacté. Ce composé mixte (très variable suivant la nature de ces protéines) étant susceptible de se fixer sur la zircone (Labbé et al., 1990) ou d'autres supports comme les aciers inoxydables (Dupeyrat et al., 1987). Fondamentalement, en effet, les protéines possèdent localement dans leur structure des charges résiduelles à plusieurs niveaux. Par exemple, des charges positives sur les atomes de calcium disséminés sur un certain nombre de sites, mais également des charges négatives au niveau des groupements carboxylates. Elles peuvent donc amorcer une fixation sur des supports très divers, surtout si l'on s'éloigne de leur point isoélectrique. Les ions calcium extérieurs viennent de toute façon se complexer avec les protéines, les carboxylates constituant des sites privilégiés (analogie avec les carbonates, qui ont leur bande principale dans la même région voisine de $1400 \mathrm{~cm}^{-1}$ ). Les premières structures de ces matériaux mixtes sont amorphes dans le cas des phosphates, et plus aisément visibles que les carbonates de calcium. Les hydroxyapatites sont les composés thermodynamiquement stables dans ces conditions, mais avec une énergie d'activation plus élevée que pour $\mathrm{CaCO}_{3}$; elles se forment au cours du temps ou sont activées par élévation de la température. Il y a bel et bien, dans ce cas, une synergie entre la formation de « dépôts » et celle de biofilm. La rigidification de ces structures, par un excès de composé inorganique cristallisé, n’intervient pas dans les premières étapes et dépend, notamment, des propriétés du composé inorganique engagé.

Dans les biofilms formés en milieu aqueux, ce sont sans doute les silicates, ou les aluminosilicates d'origine argileuse, qui jouent ce rôle de rigidification. Les résultats obtenus sur plus d'une centaine de biofilms, formés sur divers matériaux (verre, cuivre, aciers inoxydables, PVC, caoutchouc) ont montré qu'avec une alimentation à base d'eaux industrielles, de rivière ou souterraine, on obtenait toujours des aluminosilicates, quelle que soit la turbidité de l'eau utilisée. Il semble bien que les développements bactériens, révélés en infrarouge par les bandes amides I et II, soient facilités par la présence de ces composés d'origine argileuse. En retour, les développements bactériens doivent contribuer au piégeage de ces aluminosilicates. Cette hypothèse est confortée par ce que l'on peut observer dans le domaine de l'assainissement. Il est bien connu (Moletta et al., 2007) que l'adjonction de smectites colloïdales est utilisée pour améliorer le fonctionnement des fosses septiques 
en assainissement individuel. Par ailleurs, on a pu montrer récemment (Hui et al., 2008, 2010), qu'il y avait, pour les biofilms formés dans les eaux destinées à la consommation humaine, une corrélation entre la quantité de matières organiques liées à l'activité biologique et la quantité d'aluminosilicates déposés. Ce qu'on observe avec l'usage des dentifrices est tout à fait comparable, sauf que la cinétique de formation est considérablement accélérée par les charges minérales de ces produits.

\section{CONCLUSION}

L'analyse par spectrométrie infrarouge des biofilms, qui est une méthode simple, rapide à mettre en œuvre (une demi-heure par spectre), à forte sensibilité, permet d'obtenir des informations qualitatives et quantitatives sur les composés des biofilms, aussi bien organiques qu'inorganiques ; que ces derniers soient amorphes, bien ou mal cristallisés (dans ce cas, on peut évaluer un taux de cristallinité).

Dans les eaux usées évacuées des lavabos domestiques, les caractérisations effectuées ont bien confirmé que des biofilms pouvaient être formés très rapidement, en quelques dizaines de jours.

Cette activité biologique n'induit pas seulement des dépôts organiques, mais aussi des produits minéraux parmi lesquels on retrouve toujours des aluminosilicates amorphes, mais aussi, quelquefois, du carbonate de calcium sous forme de calcite ou d'aragonite. Ainsi, dans le cas de biofilms mélangés à des produits de corrosion, les bandes d'absorption caractéristiques des protéines à 1550 et $1650 \mathrm{~cm}^{-1}$ sont souvent masquées, les aluminosilicates pourraient alors servir de marqueurs révélant la présence d'activité biologique dans le processus de corrosion et d'encrassement. Or ces processus vont eux aussi être conditionnés par la concentration, dans le biofilm, de ces minéraux. Ainsi, en présence de biofilm, il arrive que la précipitation de $\mathrm{CaCO}_{3}$ soit inhibée, à cause de la présence de silicates et/ou phosphates. C'est ce qui est vérifié pour les biofilms étudiés ci-dessus.

Enfin, ces études doivent, bien entendu, être couplées avec les méthodes classiques de caractérisation des microorganismes présents par culture ou PCR.

\section{RÉFÉRENCES}

Costerton J.W., 2004. A Short History of the Development of the Biofilm Concept. In : Microbial Biofilms, Ghannoum M.A. et O’Toole G. (éds.), ASM Press, Washington, 4-19.

Daufin G. et Labbé J.P., 1998. Equipment fouling in the dairy application: problem and pretreatment. In: Amjad Z. (éd.), Calcium phosphates in biological and industrial systems, Kluwer Academic Publishers, chapitre 19, 437-463.

Dupeyrat M., Labbé J.P., Michel F., Billoudet F. et Daufin G., 1987. Mouillabilité et interactions solide-liquide dans l'encrassement de divers matériaux par du lactosérum et du lait. Lait, 67, 465-486.

Hiernaux P., 2005. Contribution de la fraction minérale des eaux au développement et à la structure des biofilms. Thèse de Doctorat de l'Université de Poitiers, France.

Hui F., Palayodan E., Quilichini B. et Lédion J., 2008. Analyse des composants inorganiques de biofilms par spectrométrie d'absorption infrarouge, J.I.E. 2008, Poitiers, Tome 2, 70-170-10. 
Hui F., Husson G.-P., Hochet B., Rebouté G. et Lédion J., 2010. Caractérisation de biofilms par spectrométrie d'absorption infrarouge. T.S.M., 7/8, 46-55.

Labbé J.P., Quémerais A., Michel F. et Daufin G., 1990. Fouling of inorganic membranes during whey ultrafiltration: an analytical methodology. J. Membr. Sci, 51, 293-307.

Labbé J.P., Lédion J. et Hui F., 2008. Infrared spectrometry for solid phase analysis: Corrosion rusts. Corr. Science, 50, 1228-1234.

Lecomte J., 1958. Spectroscopie dans l'infrarouge, Handbuch der Physik, XXVI, SpringerVerlag, Berlin.

Moletta R., Rambaud A., Maunoir S. et Philip H., 2007. Les additifs à effet biologique dans le traitement des eaux : application à la digestion anaérobie et à la fosse septique. T.S.M., 10, 31-53.

Nyquist R.A. et Kagel R.O., 1971. IR Spectra of Inorganic Compounds, IR Spectra of Inorganic Compounds, Academic Press, New York-London.

Thomassin J.H., Hiernaux P., Filal S., Merlet N. et Legube B., 2002. Relation entre la composition des eaux et la structure des biofilms : associations minéraux/bactéries, J.I.E. 2002, Poitiers, Tome 2, 63-1-63-10. 